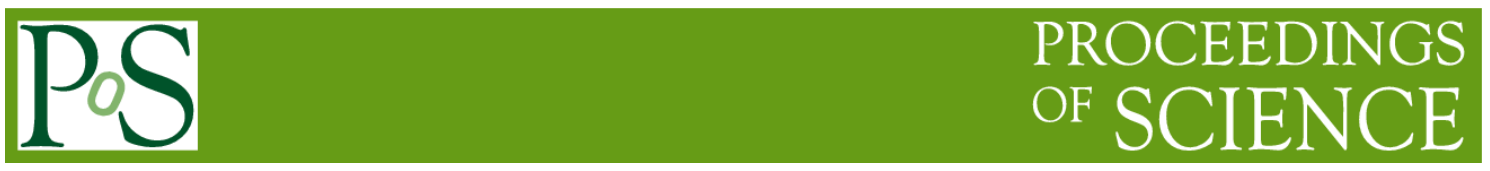

\title{
The little Hagedorn that could
}

\author{
Luciano G Moretto ${ }^{1}$ \\ Department of Chemistry, University of California, Berkeley \\ Lawrence Berkeley National Laboratory \\ 1 Cyclotron Road, Berkeley, CA 94720, USA \\ E-mail:lgomoretto@lbl.gov
}

\author{
A. C. Larsen, M. Guttormsen, S. Siem \\ Department of Physics, University of Oslo, \\ N-0316 Oslo, Norway
}

\begin{abstract}
The Hagedorn exponential mass spectrum with slope $1 / T_{H}$ was erroneously interpreted as fixing an upper limiting temperature $T_{H}$ that the system can achieve. To the contrary, such spectrum indicates a $1^{\text {st }}$ order phase transition at a fixed temperature $T_{H}$. A much lower energy example is the log linear level nuclear density below the neutron binding energy that prevails throughout the nuclear chart. We show that, for non-magic nuclei, such linearity implies a $1^{\text {st }}$ order phase transition from the pairing superfluid to an ideal gas of quasi particles.
\end{abstract}

53rd International Winter Meeting on Nuclear Physics

26-30 January 2015

Bormio, Italy

\section{${ }^{1}$ Speaker}




\section{Introduction}

The hadronic mass spectrum shows an exponential dependence that was studied and interpreted by Hagedorn with its bootstrap model ${ }^{1,2}$. Several other models ( and analogies) were suggested. Most were based on the combinatorics of self similar aggregation: of these we report the Fibonacci series as an example Other used the Euler partition applied to relativistic rotating strings which follow strict Regge trajectories. Finally the Bag $\mathrm{Model}^{3}$ in its original form gives a straightforward exponential mass spectrum (fig.1).

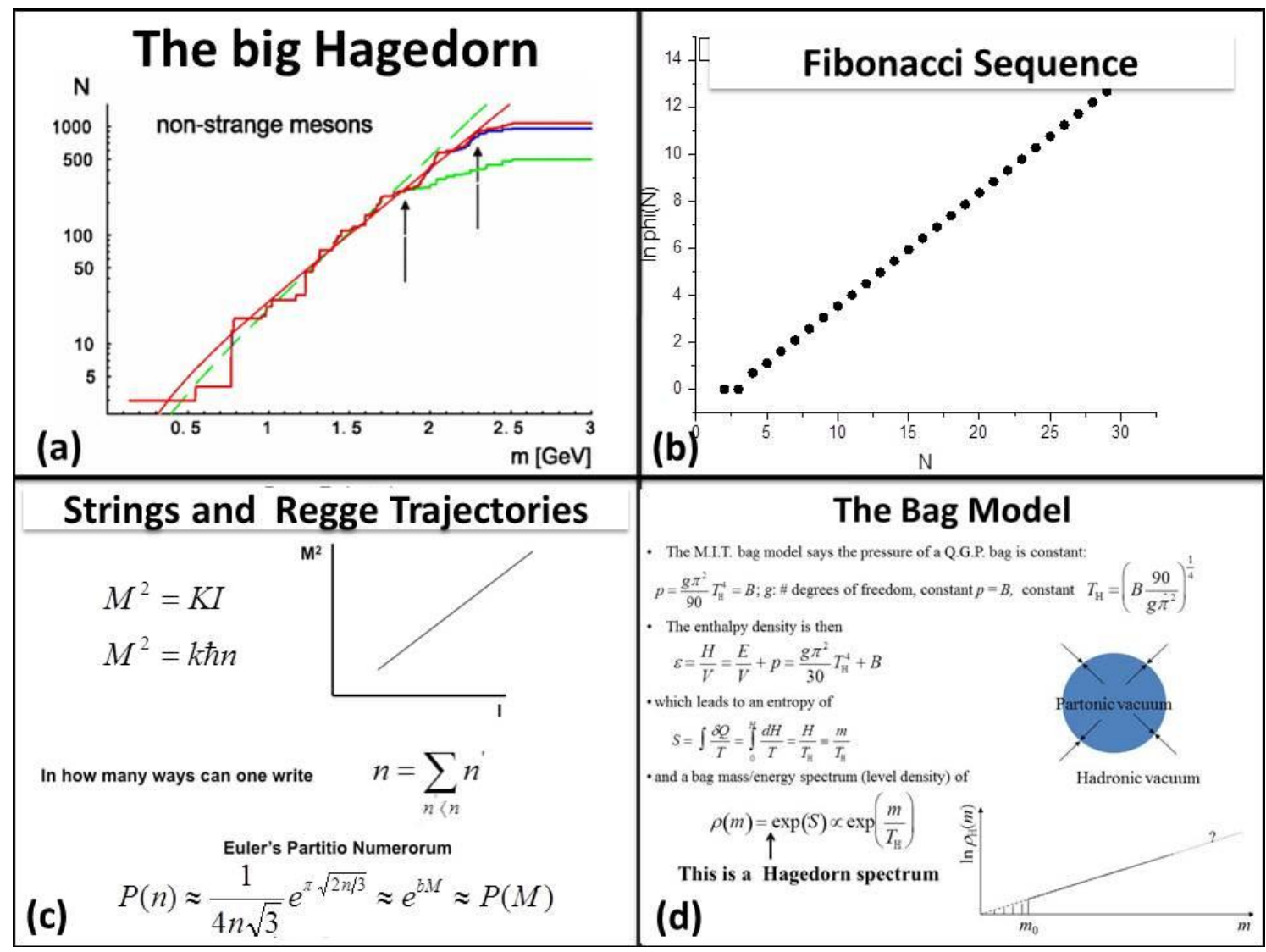

Fig.1:Experimental and theoretical examples of the Hagedorn spectra: a) An example of the hadronic Hagedorn spectrum; b) The Fibonacci sequence : an elementary example of a bootstrap model; c) The Regge trajectory of a rotating relativistic string; c) The Bag Model.

These explanations advanced over many decades justified the use of such a spectrum to obtain the partition function as follows:

Let us consider a system A with a spectrum $\rho(E)=e^{E / T_{A}}$

Thermodynamically this is a thermostat with temperature $\mathrm{T}_{\mathrm{A}}$. The level density, or spectrum, is exponential in $\mathrm{E}$ and depends only on the intrinsic "parameter" $\mathrm{T}_{\mathrm{A}}$. Let us calculate the partition function of A: 


$$
Z(T)=\int e^{E / T_{A}} e^{-E / T} d E=\int e^{-\left(1 / T-1 / T_{A}\right) E} d E=\frac{T_{A} T}{T_{A}-T} .
$$

This seems to indicate that $A$ can assume any temperature $0 \leq \mathrm{T}<\mathrm{T}_{\mathrm{A}}$. This violates thermodynamics, which requires that the only temperature possible for $\mathrm{A}$ is $\mathrm{T}_{\mathrm{A}}$. What is the trouble?

Let us consider two systems $A, B$ with level densities $\rho_{\mathrm{A}}$ and $\rho_{\mathrm{B}}$. Let the systems be thermally coupled to each other with total energy $\mathrm{E}$. We now calculate the distribution in energies between the two systems ${ }^{4}$,

$$
\rho_{T}(x)=\rho_{A}(E-x) \rho_{B}(x)
$$

Let A be a "thermostat", i.e. $\rho_{A}=e^{\varepsilon / T_{A}}$. Then

$$
\rho_{T}(x)=\exp \left(\frac{E-x}{T_{A}}\right) \rho_{B}(x)=e^{E / T_{A}} e^{-x / T_{A}} \rho_{B}(x)
$$

Let us integrate over $\mathrm{x}$

$$
\int \rho_{T}(x) d x=e^{E / T_{A}} \int e^{-x / T_{A}} \rho_{B}(x) d x=e^{E / T_{A}} Z_{B}\left(T_{A}\right)
$$

This is the thermodynamic justification of the partition function $Z_{B}\left(T_{A}\right)$ and the meaning of "implicit" thermostat. By changing "thermostat" we can change $\mathrm{T}_{\mathrm{A}}$ and the temperature of $\mathrm{B}$. Thus, every time we construct a partition function, we imply the gedanken experiment of connecting the system to a thermostat, and that this experiment is actually possible for the system we are studying. Does this always work?

To see this, let us look for the most probable value of the distribution $\rho_{\mathrm{T}}(\mathrm{x})$, which defines the equilibrium partition, by taking the log and differentiating:

$$
\begin{aligned}
\ln \rho_{T}(x) & =\ln \rho_{A}(E-x)+\ln \rho_{B}(x) \\
\partial \ln \rho_{T}(x) / \partial x & =-\partial \ln \rho_{A} / \partial x\left|+\partial \ln \rho_{B} / \partial x\right|=0 \quad \Leftrightarrow \quad 1 / T_{A}=1 / T_{B} .
\end{aligned}
$$

For this to be possible, it is necessary that $\rho_{\mathrm{A}}$ and $\rho_{\mathrm{B}}$ admit the same logarithmic derivative somewhere in the allowed range of energy x (see Fig. 2).

Usually, and always for concave functions, $\mathrm{S}(\mathrm{x})=\ln \rho(\mathrm{x})$ and $T=(\partial S / \partial x)^{-1}$ is such that $0 \leq \mathrm{T} \leq \infty$. Thus, for such systems it is possible to match derivatives for whatever value of E. Thermal equilibrium is achievable over a broad range of temperatures.

However, if $\mathrm{S}_{\mathrm{A}}(\mathrm{E})=\ln \rho_{\mathrm{A}}(\mathrm{E})$ is linear in $\mathrm{E}$, then $T_{A}=(\partial S / \partial E)^{-1}$ is a constant, independent of E. In this case, it is up to $\mathrm{B}$ to look for the value of $\mathrm{x}$ at which its logarithmic derivative matches $1 / T_{A}$. The system $A$ is a "thermostat" at $T=T_{A}$ and $B$ can only try to assume the value $\mathrm{T}=\mathrm{T}_{\mathrm{B}}=\mathrm{T}_{\mathrm{A}}$, if it can do so.

Now suppose that also $S_{B}(E)=\ln \rho_{B}(E)$ is linear in $E$ with an inverse slope $T_{B}$. This means that only if $T_{A}=T_{B}$ is equilibrium possible, and the partition function of $B, Z$ B is meaningfully defined only for $T=T_{B}$ and not for $0 \leq T \leq T_{B}$. We cannot force a temperature $T \neq T_{B}$ on a thermostat. It can only have its own intrinsic temperature $\mathrm{T}_{\mathrm{B}}$. These arguments are summarized graphically in Fig. 2. Placing systems A and B into contact will lead to a continuous heat flow from one system to the other. Thermal equilibrium is not achievable. 
Summarizing: it is permissible to calculate a system's partition function only if its $S(E)$ admits as inverse derivative value such as we want to impose through our Laplace transform. Failing that, the resulting partition function does not satisfy any thermodynamic criterion.

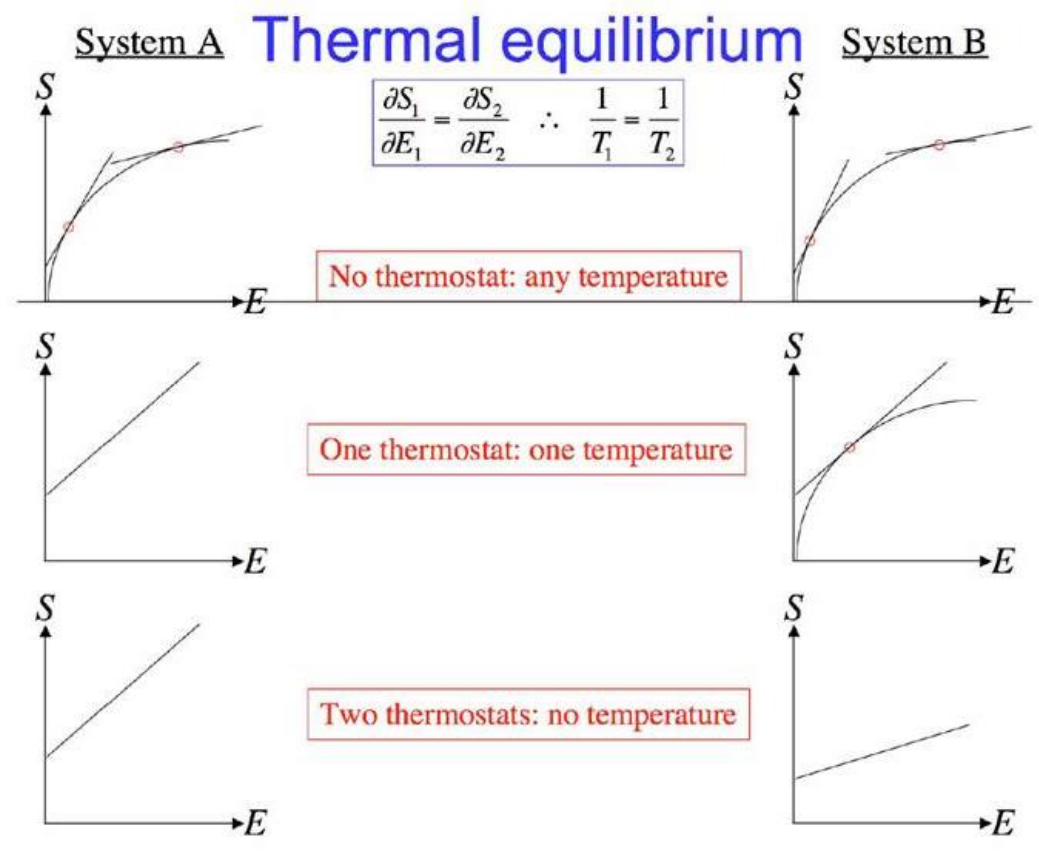

Fig. 2. Consequences of placing systems $A$ and $B$ in thermal contact when neither (top), one (middle), or both (bottom) are thermostats.

The failure to realize that the constant temperature $T_{H}$ in the Hagedorn spectrum is the one and only temperature that the system admits and that such a constant temperature represents the strongest possible microcanonical signal of a $1^{\text {st }}$ order phase transition has led to the search over many decades of alternative and possible questionable signals for the expected phase transition. From this analysis this much can be said:

1. If the mass spectrum is truly exponential it indicates a strong $1^{\text {st }}$ order phase transition ( probably hadronic to partonic)

2. All the physics of the transition is encoded in the very temperature $\mathrm{T}_{\mathrm{H}}$. Its value, near the pion mass is suggestive indeed.

\section{The little Hagedorn: first order phase transition in very low energy nuclei.}

For atomic nuclei away from closed shells and at low excitation ener-gies, the pairing force is the dominant two-body residual interaction and plays a major role together with the onebody shell-model component (the single-particle term). The presence of a gap and the compression of the quasiparticle spectrum compared to the one-body single-particle spectrum are of major importance in this discussion. For conventional superconductors, the standard BCS pairing description predicts a critical temperature/angular momentum at which the superconducting phase reverts to the normal one through a second-order phase transition. On the other hand, first-order phase transitions can arise from the BCS Hamiltonian under specific circumstances, as demonstrated in Ref. ${ }^{5}$. 
In this section, we show that a first-order, rather than a second-order, phase transition is dramatically evident in experimental nuclear level densities below neutron threshold, and that this first-order transition is, in most nuclei, indisputably related to the presence of an energy gap in the quasiparticle spectrum.

\section{- 2.1 The experimental evidence.}

A large body of high-quality nuclear level-density data are now available in literature $^{6,7,8}$. The stunning, common feature of their level densities, particularly evident for deformed, midshell nuclei, is the linear dependence of their logarithm with excitation energy. Above $\approx 2 \Delta_{0}$, where $\Delta_{0}$ is the pair-gap parameter, and up to about the neutron separation energy, they are well described by the constant-temperature expression proposed by Ericson ${ }^{9}$, and Gilbert and Cameron ${ }^{10}$ :

$$
\rho(E) \propto \exp (E / T),
$$

where $\mathrm{E}$ is the excitation energy and $\mathrm{T}$ is the constant nuclear temperature.

They found this expression to be in good agreement with the cumulative number of levels at low excitation energy, but did not provide any fundamental, quantitative explanation for this relation. Moreover, the constant-temperature expression is in striking contrast to the expected Fermi-gas behavior as first outlined by Bethe ${ }^{11}$, predicting a square-root dependence of the level density with excitation energy:

$$
\rho(E) \propto \exp (2 \sqrt{a E})
$$

where $a$ is the level-density parameter.

This experimental linear dependence of the entropy

$$
S(E) \approx \ln \rho(E)
$$

as given by Eq. (1) is the microcanonical hallmark of first-order phase transitions. Surprisingly, we may have been staring at the biggest signal yet of such a transition without seeing it. This transition is, at least for nuclei well away from closed shells, clearly related to pairing. If we, provisionally, take the constant temperature of the experimental level-density spectrum to be the BCS critical temperature, then, according to the well-known BCS relation

$$
T_{\mathrm{cr}}=\frac{2 \Delta_{0}}{3.53}
$$

we can extract the gap parameter $\Delta_{0}$ and compare it directly with that obtained from even-odd mass differences represented in the liquid-drop term

$$
\Delta_{\mathrm{BM}} \approx 12 A^{-1 / 2} \text {. }
$$

For a wide range of mass number $\mathrm{A}$, the resulting relationship between mass number and temperature using Eq. 4 is shown in Fig. 3, where the experimental constant temperatures $\mathrm{T}_{\mathrm{CT}}$ are taken from Refs. ${ }^{12,13,14}$. 


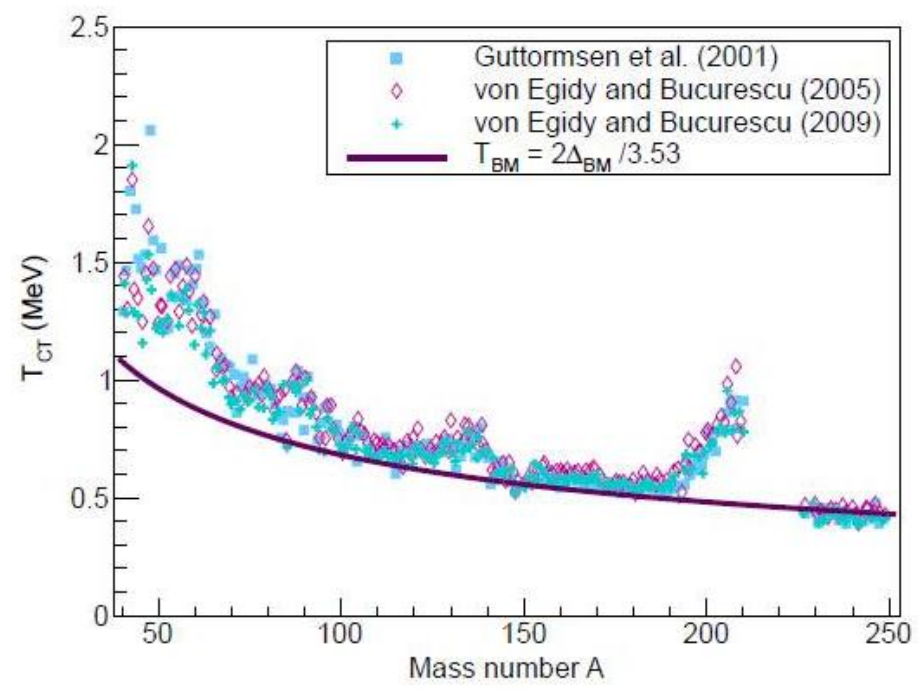

Fig 3 : (Color online) Relation between mass number $A$ and constant temnerature $T_{\mathrm{CT}}$ taken trom Refs. $\mathbf{1 2 , 1 3 , 1 4}$ for $A=40-250$, compared to $T_{\text {cr }}$ from Eq. (3) utilizing Eq (4) (dark purple line).

The close agreement in magnitude and trend is remarkable for A > 100 and away from closed shells, although the assimilation of the constant level-density temperature characteristic of a first-order transition to a critical temperature associated with a second-order transition remains to be explained. As a consequence of this observation, given the even-odd mass difference, we can predict the low-energy nuclear level densities throughout the nuclear chart for regions away from magic proton/neutron numbers. Before we embark on the explanation of this remarkable feature, let us consider another striking experimental observation: the level densities of neighboring even-even and odd-A nuclei have nearly identical slopes, as seen in Fig. 4 showing data from the rare-earth region ${ }^{15,16,17,18}$, and several actinides ${ }^{19,7}$ 

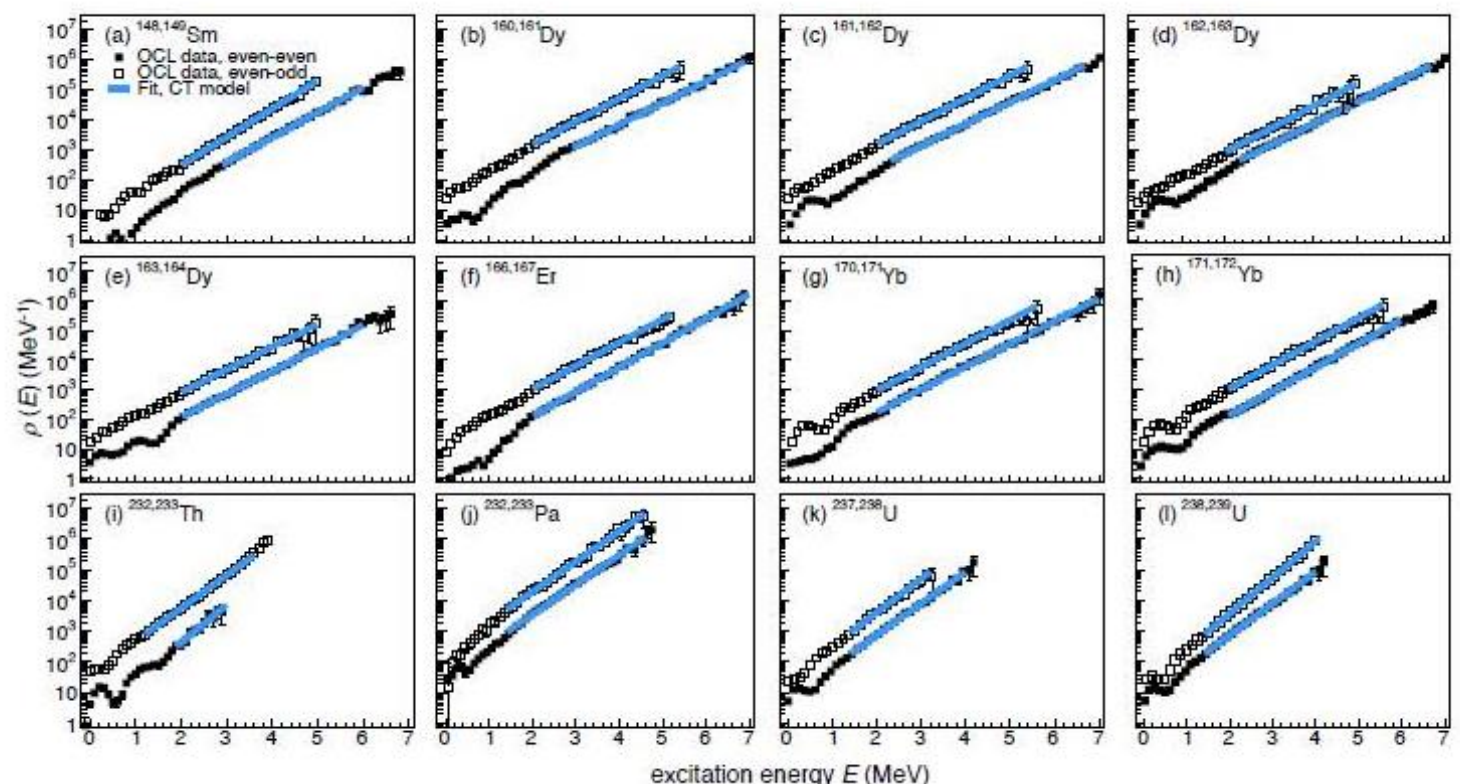

Fig 4

Experimental level densities for rare-earth and actinide nuclei measured at the Oslo Cyclotron Laboratory (OCL), with a fit of the constanttemperature model (blue line) for excitation energies above $\approx 2 \mathrm{MeV}:(\mathrm{a}){ }^{148,149} \mathrm{Sn}$ (15)
(b) ${ }^{160,161} \mathrm{Dy}$
(g) ${ }^{170,171} \mathrm{Yb}$ (18) (h) ${ }^{171,172} \mathrm{Yl}$
(16) (d) ${ }^{162,163} \mathrm{Dy}$
(16),$(e){ }^{163,164}$
(7) (j) ${ }^{232,233} \mathrm{~Pa}$
(l) ${ }^{238,239} \mathrm{U}$ (7)
(18) (i) ${ }^{232,233} \mathrm{Th}$ (7)
yy (16) f) ${ }^{166,167} \operatorname{Er}$ (17)
19) (k) ${ }^{237,238} \mathrm{U}$ (7) เnd

Therefore, the level densities of neighboring isotopes can be made to overlap by means of a horizontal shift along the excitation energy axis (see Fig. 5 and Ref. ${ }^{20}$ ). The resulting shift is constant with energy and in very good agreement with the even-odd mass difference; seeTable 1. As a consequence, locally, for any given pair of even-even and odd-A nuclei, we can calculate the common slope of the two level densities directly from the observed excitation-energy shift.

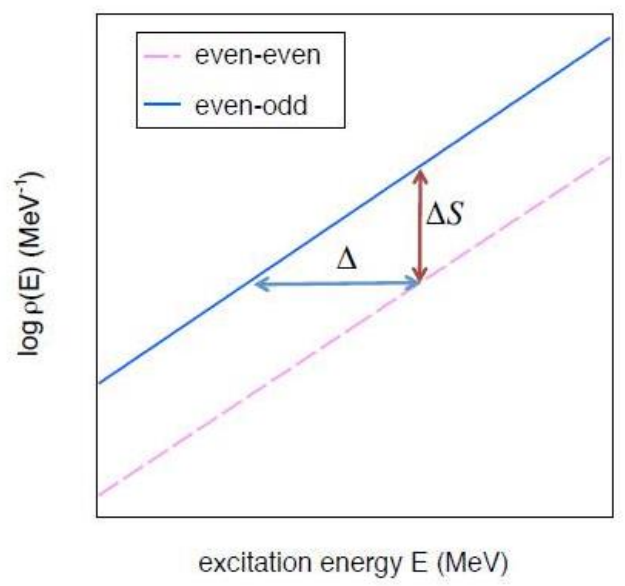

Fig 5 (Color online) Illustration of constant-temperature level densities. The experimental, horizontal shift gives the slope $\left(1 / T_{\mathrm{CT}}\right)$ through Eq. (3) and the vertical shift is related to the entropy excess $\left(S \sim \ln \rho(E), \Delta S=\ln \left[\rho_{\mathrm{eo}}(E) / \rho_{\mathrm{ee}}(E)\right]\right)$ for the quasiparticle as indicated in the figure. 
Equally intriguing is the vertical shift between the even-even-odd-A nuclear level densities, bringing the lower even-even level density on top of the higher odd-A one (see Fig. 5 and Ref. ${ }^{12}$. This difference in entropy, approximately constant throughout the energy range, can be interpreted as the entropy carried by the extra quasiparticle ${ }^{12}$. This experimental evidence alone thus suggests that as the system is excited, quasi-particles are created with a constant energy cost and carrying a constant amount of entropy, see Table 1. This theory-independent observation is a clear signature of a first-order phase transition, from a superfluid phase to an ideal gas of quasiparticles.

\begin{tabular}{|c|c|c|c|c|c|c|c|}
\hline Nuclide & $\begin{array}{c}T_{\mathrm{BM}} \\
(\mathrm{MeV})\end{array}$ & $\begin{array}{c}T_{\mathrm{CT}} \\
(\mathrm{MeV})\end{array}$ & $\begin{array}{c}T_{\text {eo }} \\
(\mathrm{MeV})\end{array}$ & $\begin{array}{c}\Delta_{\mathrm{BM}} \\
(\mathrm{MeV})\end{array}$ & $\begin{array}{c}\Delta_{\mathrm{CT}} \\
(\mathrm{MeV})\end{array}$ & $\begin{array}{c}\Delta_{\mathrm{eo}} \\
(\mathrm{MeV})\end{array}$ & $\begin{array}{c}\Delta S \\
\left(k_{B}\right)\end{array}$ \\
\hline${ }^{148} \mathrm{Sm}$ & 0.56 & $0.51(1)$ & - & 0.99 & $0.90(2)$ & - & - \\
\hline${ }^{149} \mathrm{Sm}$ & 0.56 & $0.46(1)$ & $0.51(6)$ & 0.98 & $0.81(2)$ & $0.9(1)$ & $2.0(2)$ \\
\hline${ }^{160} \mathrm{Dy}$ & 0.54 & $0.60(1)$ & - & 0.95 & $1.05(1)$ & - & - \\
\hline${ }^{161} \mathrm{Dy}$ & 0.54 & $0.58(2)$ & $0.57(6)$ & 0.95 & $1.01(3)$ & $1.0(1)$ & $1.9(2)$ \\
\hline${ }^{162} \mathrm{Dy}$ & 0.54 & $0.60(1)$ & - & 0.94 & $1.05(2)$ & - & - \\
\hline${ }^{163} \mathrm{Dy}$ & 0.53 & $0.57(2)$ & $0.51(6)$ & 0.94 & $1.00(4)$ & $0.9(1)$ & $1.9(2)$ \\
\hline${ }^{164} \mathrm{Dy}$ & 0.53 & $0.56(1)$ & - & 0.94 & $0.99(1)$ & - & - \\
\hline${ }^{166} \mathrm{Er}$ & 0.53 & $0.52(1)$ & - & 0.93 & $0.92(1)$ & - & - \\
\hline${ }^{167} \mathrm{Er}$ & 0.53 & $0.56(2)$ & $0.51(6)$ & 0.93 & $0.99(4)$ & $0.9(1)$ & $2.0(2)$ \\
\hline${ }^{170} \mathrm{Yb}$ & 0.52 & $0.57(1)$ & - & 0.92 & $1.00(1)$ & - & - \\
\hline${ }^{171} \mathrm{Yb}$ & 0.52 & $0.55(1)$ & $0.51(6)$ & 0.92 & $0.96(1)$ & $0.9(1)$ & $1.8(2)$ \\
\hline${ }^{172} \mathrm{Yb}$ & 0.52 & $0.54(1)$ & - & 0.91 & $0.95(1)$ & - & - \\
\hline${ }^{231} \mathrm{Th}$ & 0.45 & $0.41(1)$ & $0.51(11)$ & 0.79 & $0.72(2)$ & $0.9(2)$ & $2.4(4)$ \\
\hline${ }^{232} \mathrm{Th}$ & 0.45 & $0.34(1)$ & - & 0.79 & $0.60(2)$ & - & - \\
\hline${ }^{233} \mathrm{Th}$ & 0.45 & $0.40(2)$ & $0.51(11)$ & 0.79 & $0.70(2)$ & $0.9(2)$ & $2.3(4)$ \\
\hline${ }^{232} \mathrm{~Pa}$ & 0.45 & $0.44(1)$ & $0.40(6)$ & 0.79 & $0.77(2)$ & $0.7(1)$ & $1.7(2)$ \\
\hline${ }^{233} \mathrm{~Pa}$ & 0.45 & $0.45(1)$ & - & 0.79 & $0.79(2)$ & - & - \\
\hline${ }^{237} \mathrm{U}$ & 0.44 & $0.40(1)$ & $0.40(6)$ & 0.78 & $0.70(2)$ & $0.7(1)$ & $1.9(2)$ \\
\hline${ }^{238} \mathrm{U}$ & 0.44 & $0.42(1)$ & - & 0.78 & $0.74(2)$ & - & - \\
\hline${ }^{239} \mathrm{U}$ & 0.44 & $0.37(1)$ & $0.37(3)$ & 0.78 & $0.65(1)$ & $0.65(5)$ & $2.5(5)$ \\
\hline
\end{tabular}

\section{- 3 The consistency with the BCS Hamiltonian}

Now we proceed to show that all these features are consistent with the BCS theory, with due caution for the microcanonical/canonical language. For a set of uniformly spaced singleparticle levels, at the critical temperature the excitation energy is given by ${ }^{5}$

$$
E_{\mathrm{cr}}=\frac{1}{2} g \Delta_{0}^{2}+\frac{\pi^{2}}{3} g T_{\mathrm{cr}}^{2},
$$

with $g$ equal to the density of doubly degenerate single-particle states. The most probable number of quasiparticles $\mathrm{Q}_{\mathrm{Cr}}$ at $\mathrm{T}_{\mathrm{Cr}}$ is ${ }^{5}$

$$
Q_{\mathrm{cr}}=4 g T_{\mathrm{cr}} \ln 2 \text {. }
$$


By taking the ratio of these two quantities and utilizing Eq. (3), we obtain the average cost per created quasiparticle up to $\mathrm{T}_{\mathrm{cr}}$ to be

$$
\frac{E_{\mathrm{cr}}}{Q_{\mathrm{cr}}}=\Delta_{0} .
$$

This is an exact and very surprising result. Within the BCS theory, we know that at $\mathrm{T}=0$ the quasiparticle energy is $\approx \Delta_{0}$, and that $\Delta$ decreases with increasing temperature, so that $\Delta=0$ at $\mathrm{T}_{\mathrm{cr}}$.

So, how is it then possible for the energy per quasiparticle to be constant in this excitationenergy range? The explanation lies mostly in the structur of the quasiparticle energy, which reads

$$
E_{k}=\sqrt{\left(\epsilon_{k}-\lambda\right)^{2}+\Delta^{2}}
$$

where $\varepsilon_{\mathrm{k}}$ and $\lambda$ are the single-particle energy and chemical potential, respectively. As the temperature increases, $\Delta$ does indeed decrease, but within the uniform-spacing model this can be compensated by the increase of the average value of $\varepsilon_{\mathrm{k}}-\lambda$ as $\varepsilon_{\mathrm{k}}$ increases, and by the change of the underlying pairing field.

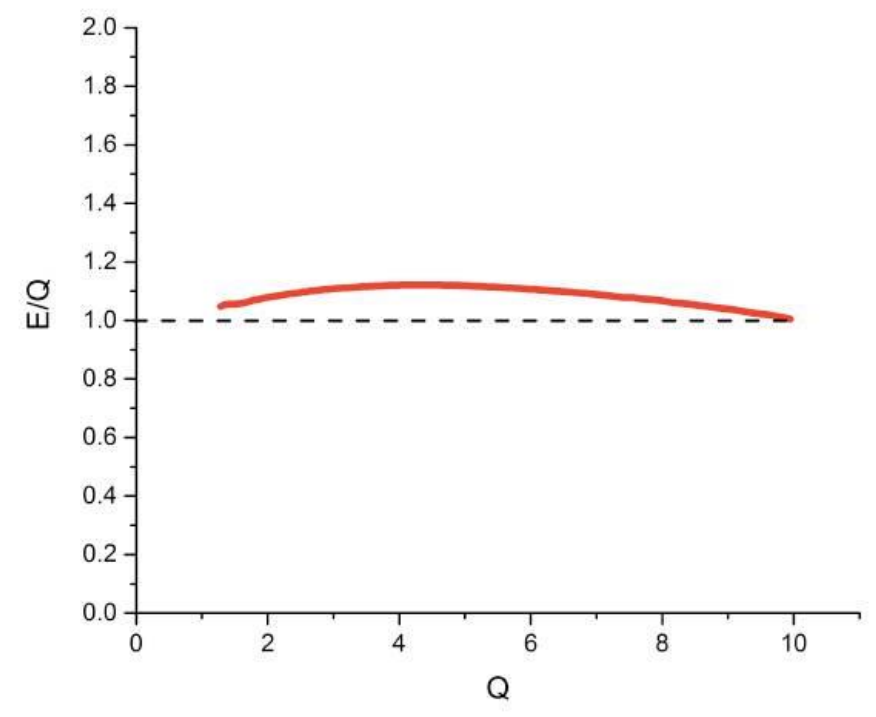

Fig 6 (Color online) Average energy per quasiparticle as a function of the most probable quasiparticle number (see text).

The assimilation of T with Tcr finds also an explanation in the BCS model. The dependence of the heat capacity upon temperature rises exponentially, and peaks at $\mathrm{T}=\mathrm{T}_{\mathrm{cr}}$, so that essentially all energy is absorbed at this temperature. Thus arise the constant temperature of the spectrum and its closeness to the BCS $\mathrm{T}_{\mathrm{cr}}$.

From the essentially constant energy $\operatorname{cost} \Delta_{0}$ per quasiparticle, it follows that the entropy per quasiparticle is

$$
\frac{\partial S}{\partial Q}=\frac{\Delta_{0}}{T_{\mathrm{cr}}}=\frac{3.53}{2}=1.77,
$$

to be compared with the empirical, vertical shift as discussed above (see Tab. 1).

Consequently, just using the energy rather than the temperature as the independent variable, we observe the progressive creation of quasiparticles, in number proportional to the energy, like the 
amount of ice melted is proportional to the absorbed heat, independent of the amount of previously melted ice. This independence, together with the constant entropy per quasiparticle, gives clear evidence of a first-order phase transition.

\section{- Conclusions}

The resulting overall picture is that of a coexistence between an underlying superfluid phase in equilibrium withan ideal vapor of almost independent quasiparticles. This picture comes about mostly by a shift of perspective, from the canonical to microcanonical approach or from temperature to energy as the independent variable. Also,the experimental availability of entropy versus energy for even-even and odd-A or odd-odd nuclei allows one to observe the very pictorial feature of gap superconductors and gap-less superconductors in mesoscopic systems. We expect these features and their interpretation to be very relevant for other mesoscopic systems, such as hadronic systems and superconductive clusters differing by just one electron.

\section{References}

${ }^{1}$ R.Hagedorn, Suppl.Nuovo Cimento 3,147 (1965)

${ }^{2}$ R.Hagedorn and J. Ranft, , Suppl.Nuovo Cimento 6,169 (1968)

${ }^{3}$ A.Chodos et al. Phys. Rev. D9, 3471 (1974(

${ }^{4}$ L.G.Moretto, K.A. Bugaev, J.B.Elliot and L.Phair, Europhys. Lett. 76, 402 (2006)

${ }^{5}$ L. G. Moretto, Nucl. Phys. A 243, 77 (1975).

${ }^{6}$ Data measured at the Oslo Cyclotron Laboratory with the Oslo method, references and data can be found at http://www.mn.uio.no/fysikk/english/research/about/infrastructure/OCL/nuclear-physicsresearch/compilation

${ }^{7}$ Data from particle-evaporation experiments, references and data can befound at http://inpp.ohiou.edu/ voinov/index.html

${ }^{8}$ A. V. Voinov, B. M. Oginni, S. M. Grimes, C. R. Brune, M. Guttormsen,A. C. Larsen, T. N. Massey, A. Schiller, and S. Siem, Phys. Rev. C 79, 031301(R) (2009).

${ }^{9}$ T. Ericson, Nucl. Phys. 11, 481 (1959).

${ }^{10}$ A. Gilbert and A. G. W. Cameron, Can. J. Phys. 43, 1446 (1965).

${ }^{11}$ H. A. Bethe, Phys. Rev. 50, 332 (1936).

12 M. Guttormsen et al., Phys. Rev. C 63, 044301 (2001).

${ }^{13}$ T. von Egidy and D. Bucurescu, Phys. Rev. C 72, 044311 (2005); Phys.Rev. C 73, 049901(E) (2006).

${ }^{14}$ T. von Egidy and D. Bucurescu, Phys. Rev. C 80, 054310 (2009).

${ }^{15}$ S. Siem et al., Phys. Rev. C 65, 044318 (2002).

${ }^{16}$ M. Guttormsen et al., Phys. Rev. C 68, 064306 (2003).

${ }^{17}$ E. Melby et al., Phys. Rev. C 63, 044309 (2001).

${ }^{18}$ U. Agvaanluvsan et al., Phys. Rev. C 70, 054611 (2004).

${ }^{19}$ M. Guttormsen et al., Phys. Rev. C 89, 014302 (2014).

${ }^{20}$ M. Guttormsen et al., Phys. Rev. C 61, 067302 (2000). 